\title{
The Watchman FLX - a new device for left atrial appendage occlusion - design, potential benefits and first clinical experience
}

\author{
Marek Grygier ${ }^{1}$, Anna Olasińska-Wiśniewska ${ }^{1}$, Aleksander Araszkiewicz ${ }^{1}$, Olga Trojnarska ${ }^{1}$, \\ Anna Babicz-Sadowska², Maciej Lesiak ${ }^{1}$ \\ ${ }^{1}$ Chair and $1^{\text {st }}$ Department of Cardiology, Poznan University of Medical Sciences, Poznan, Poland \\ ${ }^{2}$ Cardiology Department, Copper Health Center, Lubin, Poland
}

Adv Interv Cardiol 2017; 13, 1 (47): 62-66

DOI: https://doi.org/10.5114/aic.2017.66188

\section{Introduction}

Although convincing data showed that left atrial appendage (LAA) occlusion could be used as an alternative to oral anticoagulants, LAA occlusion has emerged as a common procedure for stroke prevention in patients with atrial fibrillation and absolute or relative contraindications for oral anticoagulation in many European centers, based on current recommendations of the European Society of Cardiology. The efficacy and safety of the current generation Watchman LAA Closure System was established in two large randomized clinical trials (PROTECT AF, PREVAIL [1, 2]) and several prospective registries (CAP, ASAP, EWOLUTION [3-5]).

\section{Device description}

The Watchman FLX (Boston Scientific) is the newest generation of LAA closure devices, which has been available since November 2015 in Europe, after CE mark approval. It has several new features compared to the previous generation of Watchman. The Watchman FLX device is available in 5 sizes $(20,24,27,31$ and $35 \mathrm{~mm})$ for ostia measuring from $15 \mathrm{~mm}$ to $32 \mathrm{~mm}$ in width and therefore can treat both smaller and larger LAA ostia compared to the previous generation of Watchman. A reduced device length enables implantation even in more shallow LAAs.

The Watchman FLX device is a self-expanding nitinol frame structure with fixation anchors and a permeable polyester (PET) fabric cover facing the LAA. The proximal face is flat with a reduced, minimal area of metal screw facing the left atrium to encourage endothelialisation and reduce post-implant thrombus formation. The nitinol 18-strut frame (compared to the 10-strut frame in the previous version) provides $80 \%$ more contact points at the LAA ostium and radially expands to maintain a proper position in the LAA. Atraumatic closed distal end has a fluoroscopic marker which enhances procedural visibility. Twelve "J" shaped fixation anchors in two rows create a proximal and distal line to aid in device stabilization in different anatomies of the LAA (10 in one row in the previous version). Intra-LAA placement avoids contact with the left atrial wall to reduce the risk of device erosion and minimizes interference with the left upper pulmonary vein and mitral valve. A greater range of compression is allowed, ranging from $10 \%$ to $27 \%$. Based on the animal data and early operators' experience from other centers (unpublished data) the Watchman FLX device has performed best when not overcompressed. Excessive oversizing is not recommended since the frame has stability at low compression due to the closed distal end geometry.

The Watchman FLX is pre-loaded in the delivery system (one size 14 French outer diameter access sheath compatible with any FLX device). It comes in two curve configurations - single and double, for different LAA orientation.

The Watchman FLX can be partially recaptured into the access sheath, re-maneuvered and repositioned either proximally, like the previous generation device, or advanced distally several times before device release due to atraumatic closed distal end using the "ball technique" which helps to find the ideal placement - this technique, quite new for Watchman operators, is well known for Amplatzer Cardiac Plug and Amplatzer Amulet left atrial appendage occluder (St. Jude Medical) implanters [6]. Full recapture is also allowed at every stage of the procedure before the final release of the device. The

\section{Corresponding author:}

Marek Grygier MD, Chair and 1 $1^{\text {st }}$ Department of Cardiology, Poznan University of Medical Sciences, 1/2 Długa St, 61-848 Poznan, Poland, phone: +48 501066 432, e-mail: mgrygier@wp.pl

Received: 17.11.2016, accepted: 20.12.2016. 
Table I. Main differences between current generation Watchman and Watchman FLX LAA Closure System

\begin{tabular}{lcc} 
Parameter & Watchman & Watchman FLX \\
\hline Sizes & $\begin{array}{c}21,24,27,30 \text { and } 33 \mathrm{~mm} \text { to treat ostia measuring } \\
\text { from } 17 \mathrm{~mm} \text { to } 30.5 \mathrm{~mm} \text { in width }\end{array}$ & $\begin{array}{c}20,24,27,31 \text { and } 35 \mathrm{~mm} \text { to treat ostia measuring } \\
\text { from } 15 \mathrm{~mm} \text { to } 32 \mathrm{~mm} \text { in width }\end{array}$ \\
\hline Design of distal end & Open distal end & $\begin{array}{c}\text { Atraumatic closed distal end with a fluoroscopic } \\
\text { marker }\end{array}$ \\
\hline Anchors & 10 in one row & 12 in two rows \\
\hline Struts in frame & 10 & 18 \\
\hline Sheath & 14 Fr (all sizes) & 14 Fr (all sizes) \\
\hline Recommended compression & $8-20 \%$ & $10-27 \%$ \\
\hline Proximal facing screw & Recessed screw hub & Flat and recessed screw hub
\end{tabular}

key feature changes of Watchman FLX in comparison to Watchman are presented in Figure 1 and Table I.

\section{Study description}

We aimed to collect the first data regarding implantation techniques, procedural success, complications and patient outcomes in a single-center all-comers registry summarizing initial experience with the new Watchman FLX LAA occluder.

The study group - to our knowledge the largest one in Europe - comprised 19 patients (9 female, 10 male) scheduled for LAA occlusion with the Watchman FLX. The

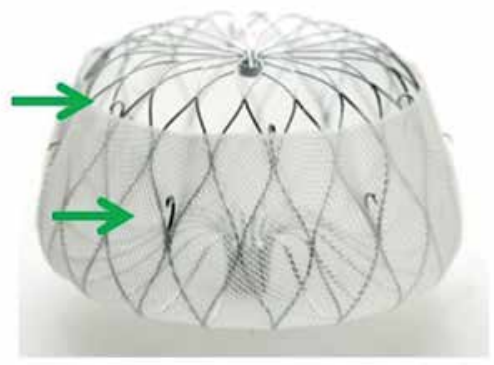

WATCHMAN FLX $27 \mathrm{~mm}$

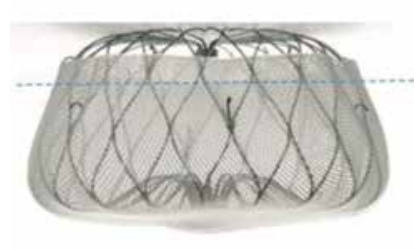

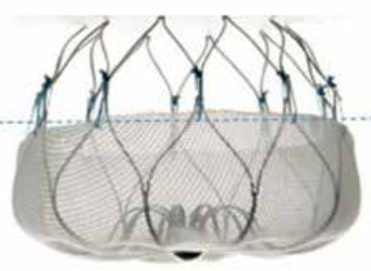

WATCHMAN $27 \mathrm{~mm}$

Figure 1. Graph representing key feature changes of the Watchman FLX in comparison to the previous Watchman device - an 18-strut frame (vs. 10 struts for Watchman), closed distal end with a fluoro marker, reduced device length, two rows of anchors (six in each row - arrows), and more PET fabric that extends down to the distal row of anchors demographic and clinical details of the study group are presented in Table II.

All procedures were performed under general anesthesia with vascular access obtained from the femoral vein. Transseptal puncture was performed under transoesophageal echocardiography (TEE). Then, the Watchman access sheath was advanced over a stiff guidewire into the left upper pulmonary vein and then repositioned to the LAA over the pigtail catheter. The LAA morphology was then carefully analyzed in both angiograms and TEE for proper device sizing. The Watchman delivery system was prepared, inserted into the access sheath, and advanced under fluoroscopic guidance. The Watchman FLX device was then deployed into the LAA using the standard technique of slowly removing the access sheath. The appropriate position was confirmed via fluoroscopy and TEE. If the position was suboptimal, the Watchman FLX could be repositioned several times both proximally, as with the previous generation device, or more distally

Table II. Demographic and clinical details of study group

\begin{tabular}{ll} 
Age & Mean: $72.6 \pm 9.2$ years (min: 61; max: 88) \\
\hline Risk scores & CHA $_{2}$ SS $_{2}$ VASC $5 \pm 1.7$ \\
& HAS-BLED $3.8 \pm 0.9$ \\
\hline Gender & 9 female \\
& 10 male \\
\hline Type of atrial & Paroxysmal, $n=13(68.4 \%)$ \\
fibrillation & Persistent, $n=6(31.6 \%)$ \\
\hline $\begin{array}{l}\text { Contraindication } \\
\text { for OAC }\end{array}$ & Bleeding, $n=16(84.2 \%):$ \\
& - intracranial, $n=2(10.5 \%)$ \\
& - gastrointestinal, $n=9(47.4 \%)$ \\
& - eye vitreous body, $n=2(10.5 \%)$ \\
& - urinary tract/pelvis, $n=2(10.5 \%)$ \\
& Strocurrent epistaxis, $n=1(5.3 \%)$ \\
& $n=6(31.6 \%)$ \\
\hline Diabetes & $n=10$ (52.6\%) \\
\hline Previous stroke & $n=2(10.5 \%)$ \\
\hline TAVI in the past & \\
\end{tabular}


using the "ball technique". The standard PASS criteria for device release was then checked (position - device is distal to or at the ostium of the LAA; anchor - fixation barbs engaged/device is stable; size - device is compressed 10$27 \%$ of original size; seal - device spans ostium, all lobes of LAA are covered). The tug test was then carried out to confirm the stability before final device release. The reverse tug test was also performed on several occasions by pushing the control wire distally after full deployment of the device - it helped better engage fixation barbs to LAA walls and, based on our experience, is useful when minimal gaps are visible after initial device placement the reverse tug test should be always followed by the standard tug test to confirm stability of the device.
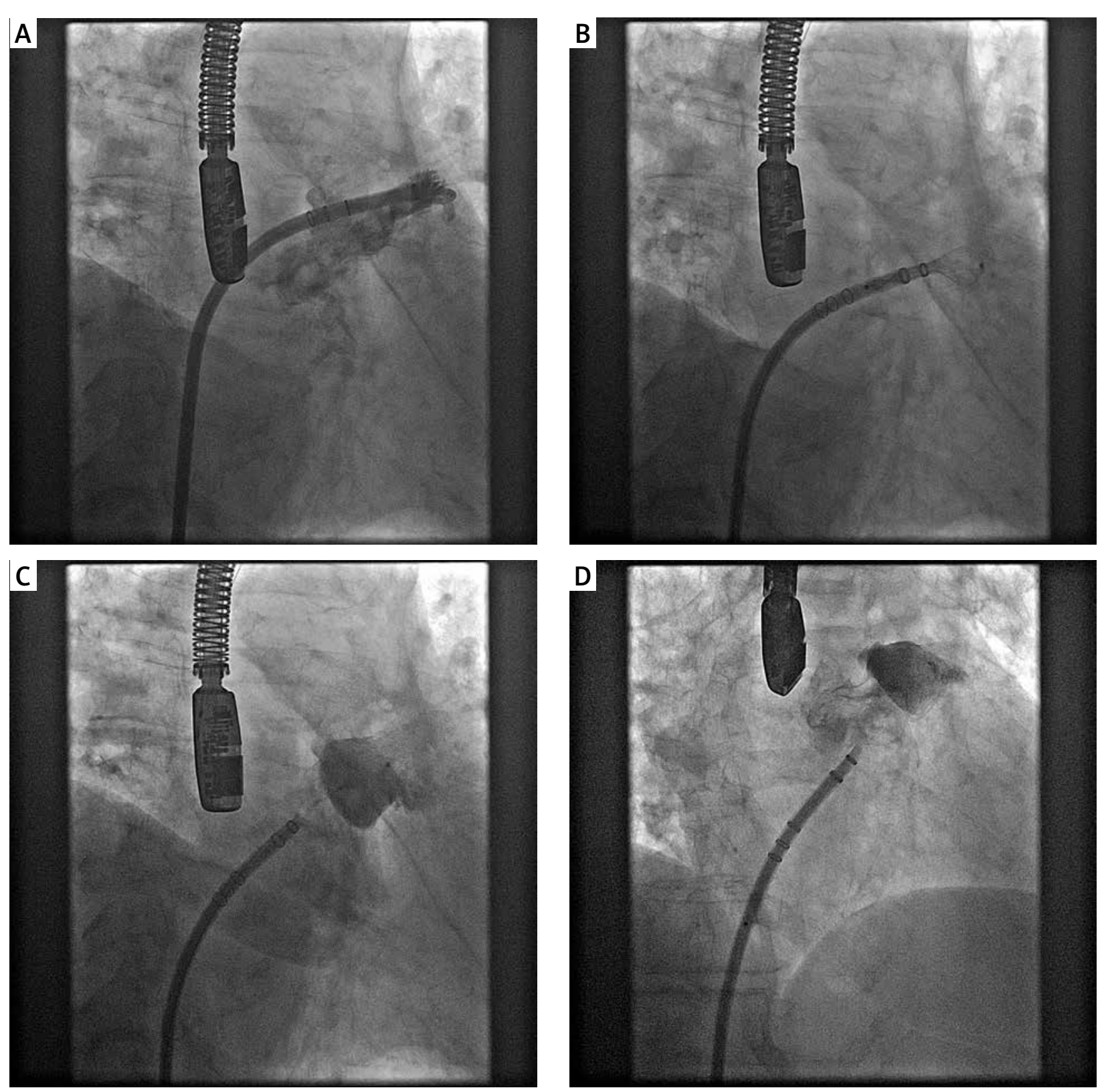

Figure 2 A-D. Angio views showing different stages of closure of LAA with Watchman FLX
The procedure was successful in 18 out of 19 patients. The first release final position was satisfactory in 4 (21\%) patients. Partial recaptures (from 1 to 10 per patient) were necessary to achieve optimal position in the remaining 15 (79\%) patients. Full recaptures (from 1 to 2 ) were necessary in 3 patients - in these cases of "chicken-wing" morphology it was easier to put the device more distally after deeper Watchman delivery system engagement over the pigtail catheter than using the "ball technique" - no device size changes of Watchman FLX were however required during the procedure. The newly available "ball technique" was used in 16 (84\%) cases (Figures 2 A-D, 3 A-D).

In 1 patient with very challenging anatomy (two large lobes extending from the very short neck in different di- 

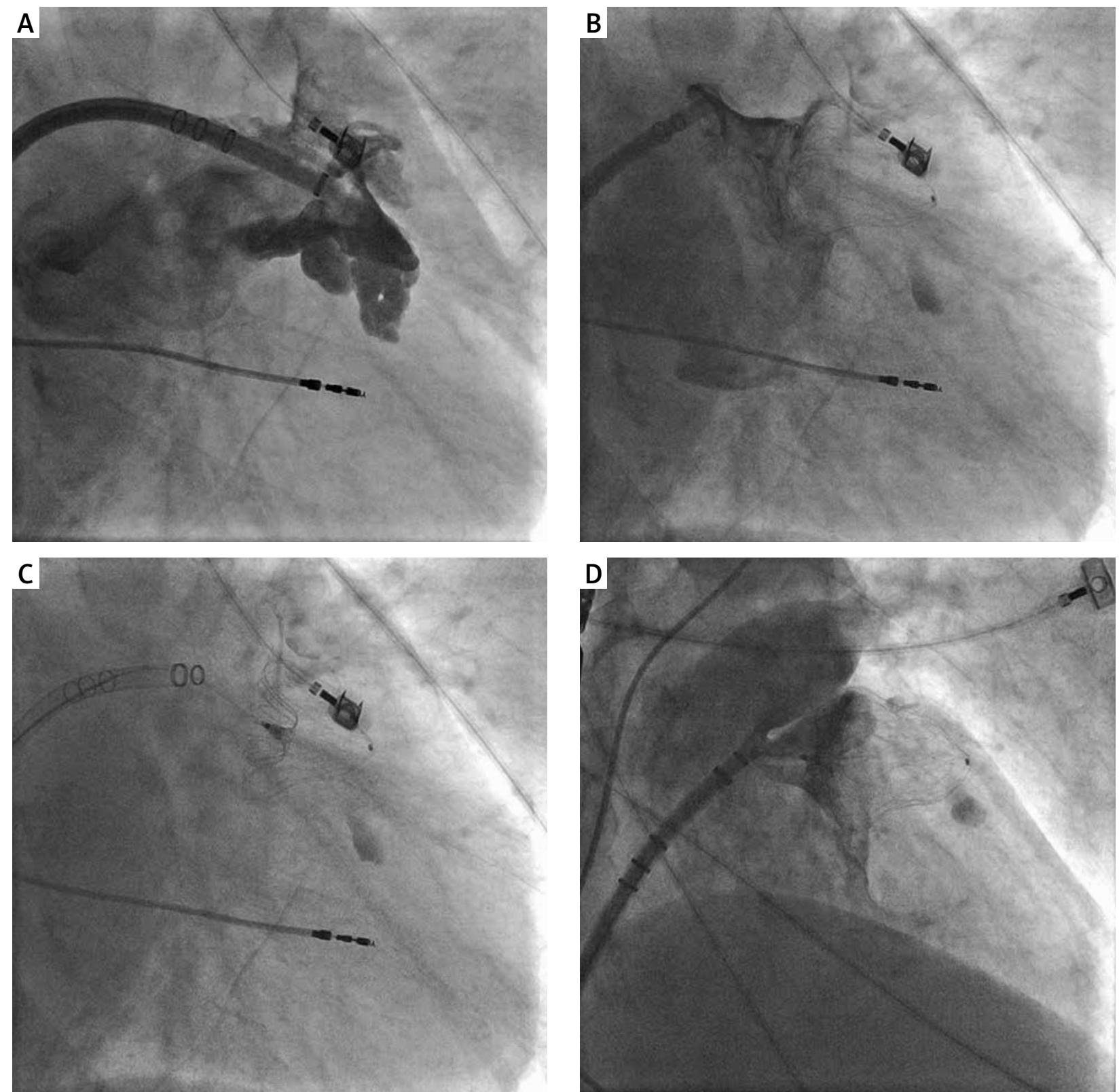

Figure 3 A-D. Angio views showing closure of very challenging LAA with Watchman FLX with reverse tug test shown in Figure $3 \mathrm{C}$

rections) it was not possible to obtain a stable position with closure of both lobes with a single Watchman FLX 24. The device was changed to the previous generation Watchman 27, but the stability was even worse. Finally the Watchman FLX 24 was implanted, but with a gap around the device of 5-8 $\mathrm{mm}$ - we decided to continue the treatment with NOAC.

The final mean Watchman FLX size was $25.4 \pm 4.6 \mathrm{~mm}$ with compression of $14.1 \pm 4.7 \%$. Mean procedure time was $46.6 \pm 16.1 \mathrm{~min}$. No device embolization, periprocedural stroke or pericardial effusions were observed in this cohort. A small gap (leakage of less than $5 \mathrm{~mm}$ ) was observed in 1 patient.
All patients except the one mentioned above were switched to dual antiplatelet therapy for at least 3 months (preferably 6 months) with the suggestion to remain on aspirin indefinitely.

Routine clinical follow-up with TEE assessment was scheduled 6-8 weeks after the procedure. We did not observe any serious adverse events. There was no thrombus on the device. Large leaks (5-8 $\mathrm{mm}$ ) were observed in 1 patient mentioned above. In two patients small leaks (1-2 $\mathrm{mm})$ around the device were observed - one in which it was observed immediately after implantation and the other one where the new leak was due to the distal device displacement within the left atrial appendage. 


\section{Conclusions}

Initial periprocedural and short follow-up data from single-center experience seem to show the new Watchman FLX occluder to be safe and effective regarding LAA occlusion. Although we observed good results, the performance and safety of the Watchman FLX will need to be confirmed in larger series of patients from other centers.

\section{Limitations}

At the end of March 2016, Boston Scientific, the manufacturer of the Watchman FLX, decided to withdraw all devices from the European market due to a higher-than-anticipated device embolization rate of $3.8 \%$ (3 acute embolizations and 5 embolizations seen post-discharge) out of 209 implants (unpublished data) coming from several institutions in which physicians with extensive clinical experience with the original Watchman device implanted the new device - the Watchman FLX. As stated, Boston Scientific has decided to pursue a design enhancement maintaining key benefits of WATCHMAN FLX such as distal redeployment, enhanced ostial seal and improved implanter control with the main goal of improving the overall device stability to allow for a more forgiving placement at the ostium. So our positive data from single-center experience, despite being the biggest such study group in Europe, should be confirmed in larger series of patients.

\section{Conflict of interest}

Marek Grygier - proctor for Watchman, research grants, Advisory Board Member, other authors declare no conflict of interest.

\section{References}

1. Reddy VY, Doshi SK, Sievert H, et al. Percutaneous left atrial appendage closure for stroke prophylaxis in patients with atrial fibrillation: 2.3-year follow-up of the PROTECT AF Trial. Circulation 2013; 127: 720-9.

2. Holmes DR Jr, Kar S, Price MJ, et al. Prospective randomized evaluation of the Watchman Left Atrial Appendage Closure device in patients with atrial fibrillation versus long-term warfarin therapy: the PREVAIL trial. J Am Coll Cardiol 2014; 64: 1-12.

3. Reddy VY, Holmes D, Doshi SK, et al. Safety of percutaneous left atrial appendage closure: results from the Watchman Left Atrial Appendage System for Embolic Protection in Patients with AF (PROTECT AF) clinical trial and the Continued Access Registry. Circulation 2011; 123: 417-24.

4. Reddy VY, Möbius-Winkler S, Miller MA, et al. Left atrial appendage closure with the Watchman device in patients with a contraindication for oral anticoagulation: the ASAP study. J Am Coll Cardiol 2013; 61: 2551-6.

5. Boersma LVA, Schmidt B, Betts T, et al. Implant success and safety of left atrial appendage closure with the WATCHMAN device: peri-procedural outcomes from the EWOLUTION registry. Eur Heart J 2016; 37: 2465-74.
6. Lam SCC, Bertog S, Gafoor S, et al. Left atrial appendage closure using the amulet device: an initial experience with the second generation amplatzer cardiac plug. Cathet Cardiovasc Interv 2015; 85: 297-303. 